\title{
Relapse of Uterine Smooth Muscle Tumor of Uncertain Malignant Potential: A Case Report with a Review of the Literature
}

\author{
Sassi I, Naija L, Ghalleb M, Chabchoub A, Jaidane O, Hechiche M, Slimane M, Rahal K \\ Surgery Department, Salah Azaiez Institute, Tunis Tunisia
}

\begin{abstract}
Introduction: Smooth muscle tumors of uncertain malignant potential (STUMP) are sporadic. We report a rare case of relapse of a uterine STUMP and discuss diagnostic and therapeutic issues.

Case report: A 25-year-old North African woman was treated for a uterine STUMP bymyomectomy. Four years later she consulted with pelvic pain associated with pollakiuria. Observations during the surgery led us to perform a hysterectomy and the histopathological examination confirmed the relapse of a STUMP.

Conclusion: Smooth muscle tumors of uncertain malignant potential of the uterus are rare. The surgery remains the standard protocol for the treatment. The risk of relapse is uncommon but a regular follow-up is recommended.
\end{abstract}

Key words: Uterine STUMP, Relapse, Genetic

\section{INTRODUCTION}

$\mathrm{S}$ mooth muscle tumors of the uterus are frequent and $\checkmark$ mostly benign. Some tumors may present unusual histological aspects that cannot be categorized as benign or malignant lesions. Thus, in 2003, the WHO classified these tumors as smooth muscle tumors of uncertain malignant potential (STUMP)[1].

STUMP are rarely reported in the literature. They are sporadic, and the exact incidence rate is unknown due to the small number of studied cases. It is difficult to categorize them by imaging, particularly for voluminous tumors, such as in our case.

Through this case, we aim to report our experience and shed further light on a rare issue of a relapse of a uterine STUMP through a literature review.

\section{Case Report}

A 25-year-old unmarried North African woman whose twin sister was operated on for a benign leiomyoma of the uterus within the same year. With a personal history of mitral shrinkage and myomectomy in 2016. The anatomopathological examination concluded a smooth muscle tumor of uncertain malignant potential. The patient was lost to follow-up for four years, and in 2020 she presented with pelvic pain associated with pollakiuria, all of which had been evolving for two years.
The clinical examination found a $20 \mathrm{~cm}$ abdominal mass.

We did not perform the gynecological examination because the patient was a virgin. The rectal examination showed a substantial mobile abdominopelvic mass. An MRI showed a globular uterus increased in size, occupying almost the entire abdominopelvic cavity measuring 260x228x161mm seat of numerous uterine myomas (Figure1), the largest of which measures $188 \times 89 \mathrm{~mm}$, in heterogeneous T2 hyper-signal, hypo-signal T1, hyper-signal diffusion with restriction of ADC and heterogeneous enhancement. After multidisciplinary consultation, the decision was to perform an exploratory laparotomy which showed small uterus pushed back above the iliac bifurcation with a $60 \mathrm{~mm}$ posterior fundal mass of soft consistency, coming into contact with the iliac vessels laterally and the cecum posteriorly and pushing back the bladder (Figure 2). The two adnexa and the rest of the abdominopelvic cavity were without any anomaly.

We performed an inter-adnexal hysterectomy (Figure 3) given to these intraoperative findings, recurrence, and histological type (STUMP). The anatomopathological examination showed the presence of fasciculated architectural tumor proliferation. It was made of smooth muscle bundles intersecting at right angles. The tumor cells were elongated, presenting significant focal atypia with an estimated mitotic index of 7 mitosis/10 fields at high magnification (CFG), presence of necrosis foci. All concluded to a STUMP. After a multidisciplinary meeting, we decided to perform clinical and radiological surveillance every three months.

No sign of recurrence was seen after ten months of follow-up.

\section{DISCUSSION}

Uterine smooth muscle tumors are categorized as benign (leiomyoma) or as malignant (leiomyosarcoma). This differentiation is based on histological criteria such as tumor cell necrosis, cytological atypia, and mitotic activity of the tumor cells [2].

In the literature, only a few cases of STUMP were reported. Lanqing Huo et al. conducted the most significant review of STUMP and showed that the median age of the patients was 42 years old [3], as has been reported in similar cases [4]. 
The occurrence of STUMP at the age of 21 and its recurrence at the age of 25 characterized our case.

Most STUMP cases manifest with several non-specific symptoms such as dysmenorrhea, abnormal vaginal bleeding or discharge, sterility, pelvic pain, pelvic mass compressing the adjacent organs, or anemia [5,6,7].

There is No sufficient published data about the radiologic characteristics of STUMP in the literature [8]. Pelvic MRI can be the reference examination to distinguish benign from malignant tumors, based on data for increased signal intensity or restriction of the signal [9].

The anatomopathological examination is the definitive tool to determine the diagnosis. However, clinicopathologic features of STUMP are not completely clear. There are now three diagnostic indicators to distinguish benign from malignant smooth muscle tumors: Mitosis number, cell atypia, and tumor cell necrosis (TCN) [10]. The presence of a single criterion makes the diagnosis of STUMP, unlike leiomyosarcoma, which requires at least two of the three diagnostic indicators.

The local or locoregional recurrence rates are diversified, varying between 7 and $27 \%[4,11,12]$. In case of relapse, STUMP may occur late, after a median of 51 months [13], and its incidence is not affected by the type of surgical intervention [4].

In order to identify which patients with STUMP had high recurrent risk, several studies have suggested the use of Immuno-histo-chemistry (IHC) by studying markers such as epithelial growth factor receptor, estrogen receptor (ER), progesterone receptor (PR), p53, p16, galectin-3, BCL-2, MIB-1, and Twist [14,15].

Lanqing Huo et al. indicated in their study that proteins $\mathrm{p} 16$, p53, and Ki-67 may have an association with STUMP recurrence [3].
In our case, due to the lack of technical means, we were unable to test this marker on the tumor cells.

Metastasis cannot be excluded in STUMP. The most common metastatic sites were the humerus, liver, and peritoneum $[16,17]$. In order to detect secondary locations, patients should get a baseline CT of the chest, abdomen, and pelvis $[4,18]$.

At the moment, there is no consensus on the surgical approach of STUMP. Myomectomy and hysterectomy may be performed by laparoscopy or laparotomy [3]. In order to preserve fertility, a myomectomy can be advised with regular clinical and radiological monitoring every six months and abdominal MRI and chest X-ray annually for the first five years $[2,19,20]$.

Otherwise, a hysterectomy is recommended because of the potential risk of recurrence [21]. In our case, despite the patient's young age and given the recurrence and intraoperative findings, hysterectomy was the treatment of choice.

This case may shed more light on a genetic factor of the STUMP occurrence. A wide-scope genetic study may be recommended to determine if there is a particular genetic mutation.

\section{CONCLUSION}

STUMP is a rare type of tumor whose diagnosis is based on an anatomopathological study.

Despite the low risk of disease recurrence or systemic spread, this tumor has a specific potential for relapse, independently of the surgical procedure used.

The patients with a diagnosis of STUMP remain on postoperative follow-up, consisting most frequently of routine physical examinations and imaging techniques twice a year for five years and after that annually.

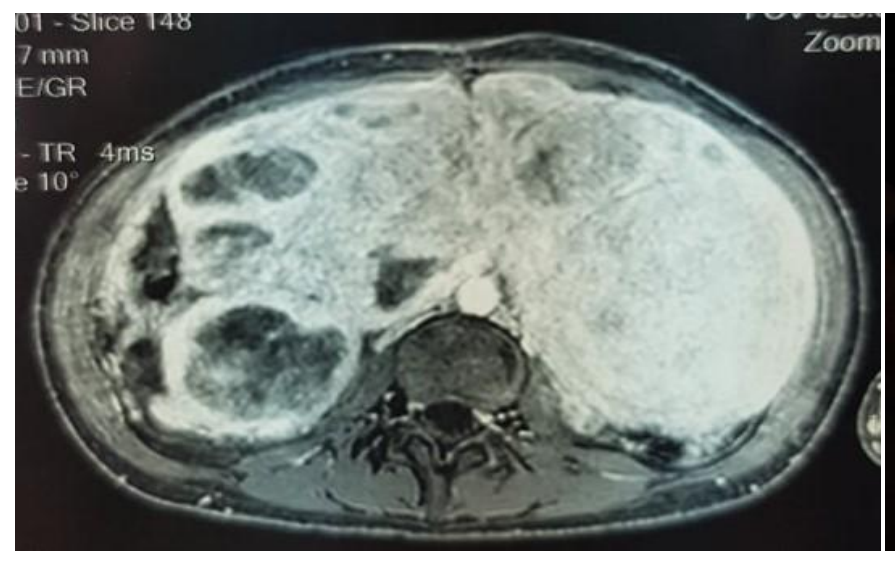

Figure 1: Globular uterus with numerous myomas showing a hyper-signal diffusion with restriction of $A D C$ and heterogeneous enhancement

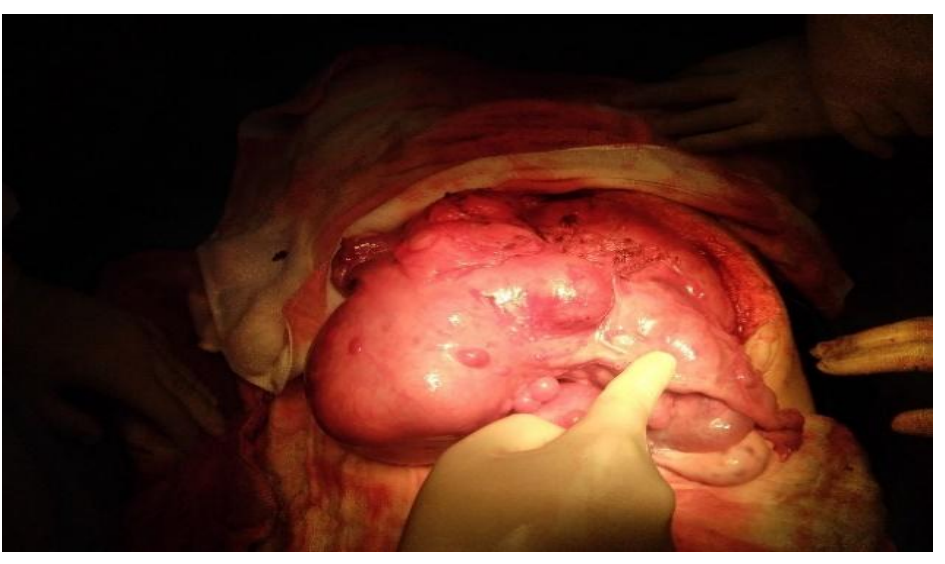

Figure 2: The uterus measuring $4 \mathrm{~cm}$ pust, $\in$ c. by the posterior fundal mass 


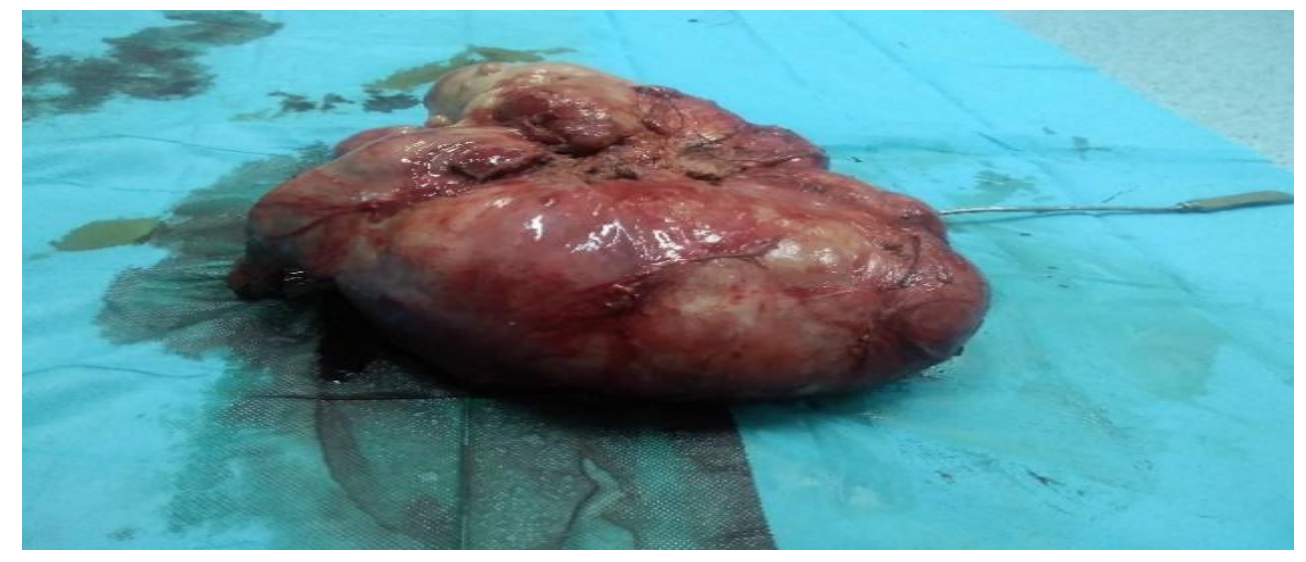

Figure 3:Inter-adnexal hysterectomy

\section{REFRENCE}

[1] Hendrickson MR, Tavassoli FA, KempsonRL, McCluggage WG, Haller U, Kubik-Huh RA. Tumours of the uterin corpus: mesenchymal tumours and related lesions. In: Tavassoli Fa, Devilee P: tumours of the breast and female genital organs: Lyon, IARC Press. 2003.

[2] Kurman RJ, Carcangiu ML, Herrington CS, Young RH. WHO classification of tumors of the female reproductive organs 4th ed WHO; 2014.

[3] Huo L, Wang D, Wang W, Cao D, Yang J, Wu M, et al. Oncologic and Reproductive Outcomes of Uterine Smooth Muscle Tumor of Uncertain Malignant Potential: A Single-Center Retrospective Study of 67 Cases. Front Oncol. 14 mai 2020;10:647.

[4] Uterine smooth muscle tumor of uncertain malignant potential: a retrospective analysis.

[5] Guntupalli SR, Ramirez PT, Anderson ML, Milam MR, Bodurka DC, Malpica A Gynecol Oncol. 2009 Jun; 113(3):324-6.

[6] Uterine Smooth Muscle Tumor of Uncertain Malignant Potential:Clinicopathologic-Sonographic Characteristics, FollowUp and Recurrence. Bacanakgil BH, Deveci M, Karabuk E, Soyman Z World J Oncol. 2017 Jun; 8(3):76-80.

[7] Uterine smooth muscle tumors other than the ordinary leiomyomas and leiomyosarcomas: a review of selected variants with emphasis on recent advances and unusual morphology that may cause concern for malignancy. Ip PP, Tse KY, Tam KF Adv Anat Pathol. 2010 Mar; 17(2):91-112.

[8] White MP, Rahimi S, Garely A, et al. . Uterine Smooth Muscle Tumors of Uncertain Malignant Potential (STUMP): Review of Pathophysiology, Classification, Diagnosis, Treatment, and Surveillance. J Healthc Commun 2017; 2: 40.

[9] Clinical application of diffusion-weighted imaging for preoperative differentiation between uterine leiomyoma and leiomyosarcoma.Sato K, Yuasa N, Fujita M, Fukushima Y Am J Obstet Gynecol. 2014 Apr; 210(4):368.e1-368.e8.

[10] Does pelvic magnetic resonance imaging differentiate among the histologic subtypes of uterine leiomyomata? Schwartz LB, Zawin M, Carcangiu ML, Lange R, McCarthy S Fertil Steril. 1998 Sep; 70(3):580-7.

[11] Uterine smooth muscle tumor analysis by comparative genomic hybridization: a useful diagnostic tool in challenging lesions. Croce S, Ribeiro A, Brulard C, Noel JC, Amant F, Stoeckle E, Devouassoux-Shisheborah M, Floquet A, Arnould L, Guyon F, Mishellany F, Garbay D, Cuppens T, Zikan M, Leroux A, Frouin
E, Duvillard P, Terrier P, Farre I, Valo I, MacGrogan GM, Chibon F Mod Pathol. 2015 Jul; 28(7):1001-10.

[12] Molecular analyses of 6 different types of uterine smooth muscle tumors: Emphasis in atypical leiomyoma. Zhang Q, Ubago J, Li L, Guo H, Liu Y, Qiang W, Kim JJ, Kong B, Wei JJ Cancer. 2014 Oct 15; 120(20):3165-77.

[13] Uterine smooth muscle tumors of uncertain malignant potential and atypical leiomyoma: a morphological study of these grey zones with clinical correlation. Deodhar KK, Goyal P, Rekhi B, Menon S, Maheshwari A, Kerkar R, Tongaonkar HB Indian J Pathol Microbiol. 2011 Oct-Dec; 54(4):706-11.

[14] Uterine Smooth Muscle Tumor of Uncertain Malignant Potential: Clinicopathologic-Sonographic Characteristics, Follow-Up and Recurrence. Bacanakgil BH, Deveci M, Karabuk E, Soyman Z World J Oncol. 2017 Jun; 8(3):76-80.

[15] Diagnostic value of progesterone receptor and p53 expression in uterine smooth muscle tumors. Hewedi IH, Radwan NA, Shash LS Diagn Pathol. 2012 Jan 5; 7():1.

[16] Atypical Uterine Smooth Muscle Tumors: A Retrospective Evaluation of Clinical and Pathologic Features. Maltese G, Fontanella C, Lepori S, Scaffa C, Fucà G, Bogani G, Provenzano S, Carcangiu ML, Raspagliesi F, Lorusso D Oncology. 2018; 94(1):1-6.

[17] Atkins KA, Arronte N, Darus CJ, Rice LW. The use of p16 in enhancing the histologic classification of uterine smooth muscle tumors. Am J Surg Pathol. 2008;32:98-102.

[18] Shapiro A, Ferenczy A, Turcotte R, Bruchim I, Gotlieb WH. Uterine smooth-muscle tumor of uncertain malignant potential metastasizing to the humerus as a high-grade leiomyosarcoma. Gynecol Oncol. 2004;94:818-820.

[19] Uterine smooth muscle tumor of uncertain malignant potential: A rare cause of multiple pulmonary nodules. Kotsopoulos IC, Barbetakis N, Asteriou C, Voutsas MG Indian J Med Paediatr Oncol. 2012 Jul; 33(3):176-8.

[20] Ly A, Mills AM, McKenney JK, et al. . Atypical leiomyomas of the uterus: a clinicopathologic study of 51 cases. Am J Surg Pathol 2013; 37: 643-649.

[21] Van den Bosch T, Dueholm M, Leone FP, et al. . Terms, definitions and measurements to describe sonographic features of myometrium and uterine masses: a consensus opinion from the Morphological Uterus Sonographic Assessment (MUSA) group. Ultrasound Obstet Gynecol 2015; 46: 284-298.

[22] Peeters N, Hulsbosch S, Ballaux F, Baekelandt J. Uterine smooth muscle tumors of uncertain malignant potential: analysis of diagnoses and therapies illustrated by two case reports. Eur $\mathbf{J}$ Gynaec Oncol 2016; 37: 367-373. 